\title{
Further Results on Sampled-Data Synchronization for Complex Dynamical Networks with Time-Varying Coupling Delay
}

\author{
Hea-Min Lee $\mathbb{D}^{1},{ }^{1}$ Wookyong Kwon $\mathbb{D}^{1},{ }^{1}$ Sangmoon Lee $\mathbb{D}^{2},{ }^{2}$ and Dongyeop Kang ${ }^{1}{ }^{1}$ \\ ${ }^{1}$ Smart Vehicles Laboratory, Electronics and Telecommunications Research Institute, Daegu 42994, Republic of Korea \\ ${ }^{2}$ School of Electronics Engineering, Kyungpook National University, Daegu 41566, Republic of Korea \\ Correspondence should be addressed to Sangmoon Lee; moony@knu.ac.kr
}

Received 5 September 2018; Revised 7 November 2018; Accepted 12 November 2018; Published 26 November 2018

Guest Editor: Hiroaki Mukaidani

Copyright (c) 2018 Hea-Min Lee et al. This is an open access article distributed under the Creative Commons Attribution License, which permits unrestricted use, distribution, and reproduction in any medium, provided the original work is properly cited.

\begin{abstract}
This paper deals with the sampled-data synchronization problem for complex dynamical networks (CDNs) with time-varying coupling delay. To get improved results, two-sided free-weighting stabilization method is utilized with a novel looped functional taking the information of the present sampled states and next sampled states, which can more precisely account for the sawtooth shape of the sampling delay. Also, the quadratic generalized free-weighting matrix inequality (QGFWMI), which provides additional degree of freedom ( $\mathrm{DoF}$ ), is utilized to calculate the upper limit of the integral term. Based on the novel looped functional and QGFWMI, improved conditions of stability are derived from forms of linear matrix inequalities (LMIs). The numerical examples show the validity and effectiveness.
\end{abstract}

\section{Introduction}

Complex dynamical networks (CDNs) are an attempt to model a set of interconnected dynamic properties of nodes with specific contents. For example, there are human interaction networks, ad hoc networks, secure communications, harmonic oscillations, biological systems, and chaotic systems, financial systems, social networks, and neural networks. CDNs are faced with the problems of expressing structural complexity and connection diversity at the same time. Furthermore, the dynamic characteristics of the network make it difficult to provide a solution to the real world because modeling should be done with the node's insufficient information from the network. Nevertheless, CDNs have attracted lots of attention in various fields of engineering [1-4]. Especially, the problem of synchronization has been focused by many researchers [5-7], as the synchronization of CDNs is a fundamental phenomenon. In nature, complex networks in the synchronization encounter time delay in biological and physical networks, because of the limited speed of network transmission, traffic jams, and signal propagation. The time delay is a source of degradation synchronization performance and instability, and thus complex networks with time-varying delay are of importance and generality $[8,9]$.
The design of control has been developed including pinning control [10], impulsive control [11], hybrid control [12], fuzzy adaptive output feedback control [13], and sampleddata control [5] to accomplish stable synchronization. Among these methods, sampled-data control for the synchronization of CDNs has been studied extensively with the development of digital communication since sampled-data offers many benefits in modern control systems. The advantages of sampled-data control are as follows: Firstly, the sampleddata control is more realistic than continuous control in that it can be implemented in practical systems. Secondly, in the case of the signal in the form of pulse data, the information is supplied immediately with a small outlay. Lastly, the control system for better performance is generally achieved by a sampled-data control. For continuous systems, the differentiator not only improves the existing noise but also generate additional noise. In the sampled-data system, the differential operation can be implemented without increasing noise problem. For that reason, sampled-data control was used because of these benefits: practicality, immediacy, economics, and accuracy. In sampled-data control systems, it is the main issue to design controllers that can get larger sampling interval. Increasing maximum sampling interval is very important because it not only enlarges the stable region 
but also improves performance when considered with other aspects: $H_{\infty}$ [14] and dissipativity [15].

Several criteria for CDNs with time delay are developed to derive stability conditions on sampled-data intervals. Sampled-data signals which are discontinuous at every sampling time can be treated as continuous time-varying delayed signals. In [5], the problem of sampled-data synchronization control for a class of general complex networks with time-varying coupling delay is firstly handled using Jensen's inequality found in the input delay approach. The timedependent Lyapunov functional and convex combination techniques are used in [16] to derive a less conservative condition for the sampled-data synchronization. The synchronization in memory neural networks with time-varying delays was studied in [2, 17, 18]. In [17], a sampled-data feedback controller was proposed by using the Lyapunov function theory and Jensen's inequality method to guarantee the synchronization of memristive Bidirectional Associative Memory (BAM) neural networks with leakage and two additive time-varying delays. The authors in [18] obtained less conservative results by constructing a Lyapunov function and using the stochastic differential inclusions and some inequality techniques. Recently, Wirtinger's inequality is used in [19, 20]. Also, the augmented Lyapunov function approach and Lyapunov function with triple integral have been reported in the literature $[15,21,22]$. In [23], a loopedfunctional-based approach was proposed for the stability analysis of linear impulsive systems. This approach easily formulates sampling interval result for discrete time stability using a continuous time's approach. In [24], a new looped-functional for stability analysis was proposed. This functional entirely uses the information on both interval $t_{k}$ and $t_{k+1}$, which improved stability condition. However, there are still more rooms for improvement, which motivates our research. To consider the information of sampling time at $t_{k}$ and $t_{k+1}$, the modified looped-functional is proposed by using the augmented vector for two-sided sampling time.

In this paper, enhanced results on sampled-data synchronization criteria and controller design are given for the complex dynamical networks with time-varying coupling delay. The stability and stabilization criteria are presented in forms of linear matrix inequalities (LMIs). The superiority of the proposed scheme is shown through numerical examples. The main contribution of this note is summarized as follows:

(i) Free-weighing matrices at time sequence $t_{k}$ and $t_{k+1}$ are separately employed with an additional scalar parameter in consideration of system dynamics in CDN satisfying convexity.

(ii) In order to fully consider the information of sawtooth shape sampling pattern at $t_{k}$ and $t_{k+1}$, novel looped functional is employed with augmented vectors which become zero by constructing the vector crossly aligned at each sampling time $t_{k}$ or $t_{k+1}$. Namely, the dimension of the LMI variable extends from $R^{2 \times 2}(2$ $\times 2$ dimensional Euclidean space) to $R^{4 \times 4}$. Therefore, augmented vectors provide an increased degrees of freedom (DoF) and improved results. (iii) QGFMI is firstly applied to sampled-data synchronization. QGFMI estimates the upper limit of the integral term more tightly. Thus it contributes to deriving a less conservative result.

\section{Preliminaries}

CDNs composed of $\mathrm{N}$ identical coupled nodes with $\mathrm{n}$ dimensional dynamics are described as follows:

$$
\begin{aligned}
\dot{x}_{i}(t)= & f\left(x_{i}(t)\right)+c \sum_{j=1}^{N} G_{i j} D x_{j}(t) \\
& +c \sum_{j=1}^{N} G_{i j} A x_{j}(t-\tau(t))+u_{i}(t), \\
& \text { for } i=1,2, \ldots, N
\end{aligned}
$$

where $f(\cdot)$ is the continuous vector-valued nonlinear function, $G_{i j}$ is the outer coupling matrix from node $i$ to $j$ with weight, $\mathrm{A}$ and $D$ are the inner coupling matrix, $x_{i}(\mathrm{t})$ is the state variable of node $i, u_{i}(t)$ is input variable ef node $i$, and $c>0$ is the coupling strength. $G_{i j}$ is defined as follows:

$$
\begin{aligned}
& G_{i j}>0 \\
& \quad \text { if there is connection between node } i \text { and } j \\
& G_{i j}=0
\end{aligned}
$$

\section{if there is no connection between node $i$ and $j$}

The diagonal elements of $G_{i j}$ are denoted as $G_{i j}=$ $-\sum_{i=1, j=i}^{N} G_{i j}$ for $i=1,2, \ldots, N$. The bounded time-varying delay $\tau(t)$ satisfies

$$
\begin{gathered}
0 \leq \tau(t) \leq \tau_{M}, \\
-\mu \leq \dot{\tau}(t) \leq \mu,
\end{gathered}
$$

where $\tau_{M}$ and $\mu$ are positive known constants. Without loss of generality, the nonlinear function $f(\cdot)$ is assumed to satisfy a sector-bounded condition as

$$
\begin{aligned}
& {\left[f(x)-f(y)-W_{1}(x-y)\right]^{T}} \\
& \quad \cdot\left[f(x)-f(y)-W_{2}(x-y)\right] \leq 0,
\end{aligned}
$$

where $W_{1}$ and $W_{2}$ are matrices with appropriate dimensions. Let $r(t)$ be an unforced isolated node, $\dot{r}(t)=f(r(t))$, then the error dynamics of each dynamical system is derived as

$$
\begin{aligned}
\dot{e}_{i}(t)= & g\left(e_{i}(t), r(t)\right)+c \sum_{j=1}^{N} G_{i j} D x_{j}(t) \\
& +c \sum_{j=1}^{N} G_{i j} A x_{j}(t-\tau(t))+u_{i}(t),
\end{aligned}
$$


where $e_{i}(t)=x_{i}-r(t)$ and $g\left(e_{i}(t), r(t)\right)=f\left(x_{i}(t)\right)-f(r(t))$. Utilizing Kronecker product, the whole CDN is represented as

$$
\begin{aligned}
\dot{e}_{i}(t)= & \widehat{g}(e(t), r(t))+c(G \otimes D) e(t) \\
& +c(G \otimes A) e(t-\tau(t))+u(t)
\end{aligned}
$$

where

$$
\begin{aligned}
& e(t)=\left[\begin{array}{c}
e_{1}(t) \\
e_{2}(t) \\
\cdots \\
e_{N}(t)
\end{array}\right] \\
& e(t-\tau(t))=\left[\begin{array}{c}
e_{1}(t-\tau(t)) \\
e_{2}(t-\tau(t)) \\
\cdots \\
e_{N}(t-\tau(t))
\end{array}\right], \\
& \hat{g}(e(t), r(t))=\left[\begin{array}{c}
g\left(e_{1}(t), r(t)\right) \\
g\left(e_{2}(t), r(t)\right) \\
\ldots \\
g\left(e_{N}(t), r(t)\right)
\end{array}\right], \\
& u(t)=\left[\begin{array}{c}
u_{1}(t) \\
u_{2}(t) \\
\ldots \\
u_{N}(t)
\end{array}\right]
\end{aligned}
$$

For the given system, our objective is to design a sampleddata controller which makes the error systems converge to zero. When considering that the measurement sensor generates a signal, discretized sampled signals are only sent to the controller through the network in system topology, and

the control input can be utilized using zero order function. The control input signals using sampler are generated with a sequence of hold time $0=t_{0}<t_{1}<\ldots<t_{k}<\ldots$ where $\lim _{k \rightarrow \infty} t_{k}=\infty$ and $k$ is a positive integer. The sampling intervals are represented as

$$
t_{k+1}-t_{k} \leq h_{k} \leq h_{M}
$$

where $h_{M}$ is the maximum sampling instant. Then, the sampled-data controller is designed as

$$
u(t)=u\left(t_{k}\right)=K\left(e\left(t_{k}\right)\right), \text { for } t_{k} \leq t \leq t_{k+1} .
$$

where $k=\operatorname{diag}\left\{K_{1}, K_{2}, \ldots, K_{N}\right\}$ is the control gain matrix and $e\left(t_{k}\right)$ is the sampled error signal. The closed-loop error systems with sampled-data control are rewritten as

$$
\begin{aligned}
\dot{e}_{i}(t)= & \widehat{g}(e(t), r(t))+c(G \otimes D) e(t) \\
& +c(G \otimes A) e(t-\tau(t))+K u\left(t_{k}\right)
\end{aligned}
$$

To develop main results, useful lemmas are introduced.

Lemma 1 (Wirtinger's inequalities; [25]). For given a matrix $R>0$ and a continuous differentiable function $\omega(t)$ in $[a, b]$, the following inequality holds:

$$
\int_{a}^{b} \omega^{T}(s) R \omega(s) d s \geq \frac{1}{b-a} \eta_{1}^{T} R \eta_{1}+\frac{3}{b-a} \eta_{2}^{T} R \eta_{2}
$$

where $\eta_{1}=\int_{a}^{b} \omega(s) d s$ and $\eta_{2}=\int_{a}^{b} \omega(s) d s-(2 /(b-$ a)) $\int_{a}^{b} \int_{s}^{b} \omega(u) d u d s$

Lemma 2 (reciprocally convex combination method; [26]\}). For a given scalar $0<\theta<1$, vectors $\omega_{1}, \omega_{2}$, and matrices $M>0, N$ satisfying $\left[\begin{array}{ll}M & N \\ N & M\end{array}\right]>0$, then the following holds:

$$
\frac{1}{\theta} \omega_{1}^{T} M \omega_{1}+\frac{1}{1-\theta} \omega_{2}^{T} M \omega_{2} \leq\left[\begin{array}{l}
\omega_{1} \\
\omega_{2}
\end{array}\right]^{T}\left[\begin{array}{ll}
M & N \\
N & M
\end{array}\right]\left[\begin{array}{l}
\omega_{1} \\
\omega_{2}
\end{array}\right]
$$

Lemma 3 (QGFMI). Given matrices X, Y and positive semidefinite matrix $R$, the inequality is given by

$$
\int_{a}^{b} \omega^{T}(s) R \omega(s) d s \geq-\left[\begin{array}{l}
\eta_{0} \\
\eta_{3}
\end{array}\right]^{T}\left[\begin{array}{cc}
(b-a) X R^{-1} X^{T} & X\left[\begin{array}{ll}
I & 0
\end{array}\right] \\
* & \frac{(b-a)}{3} Y R^{-1} Y^{T}+\operatorname{sym}\left(Y\left[\begin{array}{ll}
-I & 2 I
\end{array}\right]\right)
\end{array}\right]\left[\begin{array}{l}
\eta_{0} \\
\eta_{3}
\end{array}\right]
$$

where $\eta_{0}$ is any vector and $\eta_{3}=\left[\int_{a}^{b} \omega^{T}(s) d s,(1 /(b\right.$ $\left.-a)) \int_{a}^{b} \int_{s}^{b} \omega(u)^{T} d u d s\right]^{T}$, and $\omega(t)$ is a differentiable function which is a continuous on $[\mathrm{a}, \mathrm{b}]$.

Proof. The proof of Lemma 3 is omitted as it is similar to that of [27].
Remark 4. The QGFMI is used to calculate the upper limit of integral term in the derivative of Lyapunov-Krasovskii function, which increases freedom to choose a free-selectable vector [27]. Furthermore, the new free-weighting matrix plays a vital role in filling in the diagonal element and corresponding augmented vector provides additional flexibility. 
Remark 5. The control technique using the sample value data in (9) can be applied to systems such as event-triggered communication as in $[28,29]$.

\section{Main Results}

The matrices $I_{i}=R^{11 n \times n}$ for $i=1,2, \ldots, 11$ are denoted to represent matrices composed of $(n-1)$ zero elements matrices with $i_{\text {th }}$ identity matrix. For example, $I_{1}=$ $\left[\begin{array}{lllllllllll}I & 0 & 0 & 0 & 0 & 0 & 0 & 0 & 0 & 0 & 0\end{array}\right]^{T}$ and $I_{5}=\left[\begin{array}{lllllllllll}0 & 0 & 0 & 0 & I & 0 & 0 & 0 & 0 & 0 & 0\end{array}\right]^{T}$. Moreover, the following are declared:

$$
\begin{aligned}
& \zeta(\mathrm{t})=\left[e(\mathrm{t}), \dot{e}(\mathrm{t}), e\left(t_{k}\right), \frac{1}{\left(t-t_{k}\right)}\right. \\
& \cdot \int_{t_{k}}^{t} e^{T}(\alpha) d \alpha, e\left(t_{k+1}\right), \frac{1}{\left(t_{k+1}-t\right)} \\
& \int_{t}^{t_{k+1}} e^{T}(\alpha) d \alpha, e(t-\tau(t)), e\left(t-\tau_{M}\right), \frac{1}{\tau(t)} \\
& \cdot \int_{\mathrm{t}-\tau(t)}^{t} e^{T}(\alpha) d \alpha, \frac{1}{\left(\tau_{M}-\tau(t)\right)} \\
& \left.\cdot \int_{t-\tau_{M}}^{t-\tau(t)} e^{T}(\alpha) d \alpha, \hat{g}^{T}(e(t), s(t))\right] \\
& \kappa_{1}=\left[I_{1}, I_{3}+I_{5}, I_{4}+I_{6}, I_{2}\right] \text {, } \\
& \kappa_{2}=\left[I_{1}-I_{3}, I_{1}-I_{4}\right] \text {, } \\
& \kappa_{3}=\left[I_{5}-I_{1}, I_{5}-I_{6}\right] \text {, } \\
& \kappa_{4}=\left[I_{1}-I_{7}, I_{1}+I_{7}-I_{9}, I_{7}-I_{8}, I_{7}+I_{8}-I_{10}\right], \\
& \eta_{1}=\left[I_{1}-I_{3},\left(t-t_{k}\right) I_{5},\left(t-t_{k}\right) I_{3},\left(t-t_{k}\right) I_{4}\right] \text {, } \\
& \eta_{2}=\left[I_{5}-I_{1},\left(t_{k+1}-t\right) I_{3},\left(t_{k+1}-t\right) I_{5},\left(t_{k+1}-t\right) I_{6}\right] \text {, } \\
& \eta_{1 d}=\left[I_{2}, I_{5}, I_{3}, I_{1}\right] \\
& \eta_{2 d}=\left[-I_{2},-I_{3},-I_{5},-I_{1}\right] \text {, } \\
& S_{1}(t)=\left[\begin{array}{cc}
\left(t-t_{k}\right) S_{10}-\left(t_{k+1}-t\right) S_{11} & S_{12} \\
S_{12}^{T} & S_{13}
\end{array}\right] \in R^{4 n \times 4 n}, \\
& S_{10} \in R^{n \times n} \\
& S_{11} \in R^{n \times n} \\
& S_{12} \in R^{n \times 3 n} \text {, } \\
& S_{13} \in R^{3 n \times 3 n}, \\
& S_{1 d}=\left[\begin{array}{cc}
S_{10}-S_{11} & 0_{n \times n} \\
0_{n \times n} & 0_{n \times n}
\end{array}\right], \\
& \widehat{R}=\left[\begin{array}{ll}
R & 0 \\
0 & R
\end{array}\right] \text {. }
\end{aligned}
$$

Theorem 6. For a given scalar parameter $h_{M}$ and a gain matrix $\mathrm{K}$, if there exist a positive scalar $\delta$, symmetric matrices $\mathrm{P}>0, \mathrm{Q}_{1}>0, \mathrm{Q}_{2}>0, \mathrm{R}>0, \mathrm{~T}_{1}>0$, and $\mathrm{T}_{2}>0$, any matrices $G_{1}, G_{2}, G_{3}, G_{4}, S_{1}(t), S_{2}, X_{1}, X_{2}, Y_{1}, Y_{2}$, and $\widehat{M}$, and a scalar $\delta>0$ satisfying the inequalities

$$
\begin{aligned}
& {\left[\begin{array}{ccc}
\Pi_{1, \boldsymbol{h}(\boldsymbol{t})=\mathbf{0}} & \kappa_{1} X_{2} & \kappa_{3} Y_{2} \\
* & -h_{M} T_{2} & 0 \\
* & 0 & -3 h_{M} T_{2}
\end{array}\right] \leq 0} \\
& {\left[\begin{array}{ccc}
\Pi_{1, \boldsymbol{h}(\boldsymbol{t})=\mathbf{h}} & \kappa_{1} X_{1} & \kappa_{2} Y_{1} \\
* & -h_{M} T_{1} & 0 \\
* & 0 & -3 h_{M} T_{1}
\end{array}\right] \leq 0} \\
& \\
&
\end{aligned}
$$

where

$$
\begin{aligned}
& \Pi_{1, \boldsymbol{h}(\boldsymbol{t})}=\operatorname{sym}\left\{I_{1} P I_{2}^{T}\right\}+I_{1} Q_{1} I_{1}^{T}-(1-\mu) I_{7} Q_{1} I_{7}^{T} \\
& +I_{1} Q_{2} I_{1}^{T}-I_{8} Q_{2} I_{8}^{T}+\tau_{M} I_{2} R I_{2}^{T}-\kappa_{4}\left[\begin{array}{cc}
\widehat{R} & \widehat{M} \\
\widehat{M} & \widehat{R}
\end{array}\right] \kappa_{4}^{T} \\
& +\operatorname{sym}\left\{\eta_{1} S_{1}(t) \eta_{2 d}^{T}+\eta_{1 d} S_{1}(t) \eta_{2}^{T}+\eta_{1} S_{1 d} \eta_{2}^{T}\right\} \\
& +\operatorname{sym}\left\{\left[\begin{array}{ll}
I_{1}-I_{3} & \left(t-t_{k}\right) I_{4}
\end{array}\right] S_{2}\left[\begin{array}{c}
-I_{2}^{T} \\
-I_{1}^{T}
\end{array}\right]\right. \\
& \left.+\left[\begin{array}{ll}
I_{2} & I_{1}
\end{array}\right] S_{2}\left[\begin{array}{c}
I_{5}^{T}-I_{1}^{T} \\
\left(t_{k+1}-t\right) I_{6}
\end{array}\right]\right\}+\left(t_{k+1}-t\right) I_{2} T_{1} I_{2}^{T} \\
& +\left(t-t_{k}\right) I_{2} T_{2} I_{2}^{T}+\operatorname{sym}\left\{\kappa_{1} X_{1}\left[\begin{array}{ll}
I & 0
\end{array}\right] \kappa_{2}^{T}\right. \\
& \left.+\kappa_{2} Y_{1}\left[\begin{array}{ll}
-I & 2 I
\end{array}\right] \kappa_{2}^{T}\right\}+\operatorname{sym}\left\{\kappa_{1} X_{2}\left[\begin{array}{ll}
I & 0
\end{array}\right] \kappa_{3}^{T}\right. \\
& \left.+\kappa_{1} Y_{2}\left[\begin{array}{ll}
-I & 2 I
\end{array}\right] \kappa_{3}^{T}\right\}-\delta\left[\begin{array}{ll}
I_{1} & I_{11}
\end{array}\right]\left[\begin{array}{cc}
\widetilde{W}_{1} & \widetilde{W}_{2} \\
* & 2 I
\end{array}\right]\left[\begin{array}{c}
I_{1}^{T} \\
I_{11}^{T}
\end{array}\right] \\
& +\left(t-t_{k}\right) \operatorname{sym}\left\{\left(I_{1} G_{1}+I_{2} G_{2}\right)\right. \\
& \left.\cdot\left(c(C \otimes D) I_{1}^{T}+c(C \otimes A) I_{7}^{T}+K I_{3}^{T}-I_{2}^{T}+I_{11}^{T}\right)\right\} \\
& +\left(t_{k+1}-t\right) \operatorname{sym}\left\{\left(I_{1} G_{3}+I_{2} G_{4}\right)\right. \\
& \left.\cdot\left(c(C \otimes D) I_{1}^{T}+c(C \otimes A) I_{7}^{T}+K I_{3}^{T}-I_{2}^{T}+I_{11}^{T}\right)\right\}
\end{aligned}
$$

and $\widetilde{W_{1}}=\left(\mathrm{I} \otimes W_{1} W_{2}\right)+\left(W_{1} W_{2} \otimes \mathrm{I}\right), \widetilde{W_{2}}=-I \otimes\left(W_{1}^{T}+W_{2}^{T}\right)$, then CDNs are asymptotically stable.

Proof. Construct the following Lyapunov-Krasovskii function for $\mathrm{t} \in\left[\mathrm{t}_{k}, \mathrm{t}_{k+1}\right)$ :

$$
\begin{aligned}
\mathrm{V}\left(x_{t}\right)= & V_{1}(t)+V_{2}(t)+V_{3}(t)+V_{l 1}(t)+V_{l 2}(t) \\
& +V_{l 3}(t)+V_{l 4}(t),
\end{aligned}
$$


where

$$
\begin{aligned}
& V_{1}(t)=e^{T}(t) P e(t), \\
& V_{2}(t)=\int_{t-\tau(t)}^{t} e^{T}(\alpha) Q_{1} e(\alpha) d \alpha+\int_{t-\tau_{M}}^{t} e^{T}(\alpha) Q_{2} e(\alpha) d \alpha, \\
& V_{3}(t)=\int_{t-\tau_{M}}^{t} \int_{\beta}^{t} \dot{e}^{T}(\alpha) \operatorname{Re}(\alpha) d \alpha d \beta, \\
& V_{l 1}(t)=2\left[\begin{array}{c}
e(t)-e\left(t_{k}\right) \\
\left(t-t_{k}\right) e\left(t_{k+1}\right) \\
\left(t-t_{k}\right) e\left(t_{k}\right) \\
\int_{t_{k}}^{t} e(\alpha) d \alpha
\end{array}\right]^{T} S_{1}\left[\begin{array}{c}
e\left(t_{k+1}\right)-e(t) \\
\left(t_{k+1}-t\right) e\left(t_{k}\right) \\
\left(t_{k+1}-t\right) e\left(t_{k+1}\right) \\
\int_{t}^{t_{k+1}} e(\alpha) d \alpha
\end{array}\right] \text {, } \\
& V_{l 2}(t)=2\left[\begin{array}{c}
e(t)-e\left(t_{k}\right) \\
\int_{t_{k}}^{t} e(\alpha) d \alpha
\end{array}\right]^{T} S_{2}\left[\begin{array}{c}
e\left(t_{k+1}\right)-e(t) \\
\int_{t}^{t_{k+1}} e(\alpha) d \alpha
\end{array}\right] \text {, } \\
& V_{l 3}(t)=\left(t_{k+1}-t\right) \int_{t_{k}}^{t} \dot{e}^{T}(\alpha) T_{1} \dot{e}(\alpha) d \alpha, \\
& V_{l 4}(t)=-\left(t-t_{k}\right) \int_{t}^{t_{k+1}} \dot{e}^{T}(\alpha) T_{2} \dot{e}(\alpha) d \alpha .
\end{aligned}
$$

Differentiating each LKF in equation (20) provides

$$
\begin{aligned}
\dot{V}\left(x_{t}\right)= & \dot{V}_{1}(t)+\dot{V}_{2}(t)+\dot{V}_{3}(t)+\dot{V}_{l 1}(t)+\dot{V}_{l 2}(t) \\
& +\dot{V}_{l 3}(t)+\dot{V}_{l 4}(t),
\end{aligned}
$$

where

$$
\begin{aligned}
& \dot{V}_{1}(t)=2 e(t) P \dot{e}(t)=\operatorname{sym}\left\{I_{1}^{T} P I_{2}\right\} \\
& \dot{V}_{2}(t)=e^{T}(t) Q_{1} e(t)-(1-\mu) e^{T}(t-\tau(t)) Q_{1} e(t \\
& -\tau(t))+e^{T}(t) Q_{2} e(t)-e^{T}\left(t-\tau_{M}\right) Q_{2} e\left(t-\tau_{M}\right) \\
& =I_{1} Q_{1} I_{1}^{T}-(1-\mu) I_{7} Q_{1} I_{7}^{T}+I_{1} Q_{2} I_{1}^{T}-I_{8} Q_{2} I_{8}^{T}, \\
& \dot{V}_{3}(t)=\tau_{M} \dot{e}^{T}(t) R \dot{e}(t)-\int_{t-\tau_{M}}^{t} \dot{e}^{T}(\alpha) R \dot{e}(\alpha) d \alpha \\
& =\tau_{M} I_{2} R I_{2}^{T}-\int_{t-\tau_{M}}^{t} \dot{e}^{T}(\alpha) R \dot{e}(\alpha) d \alpha, \\
& \dot{V}_{l 1}(t)=2\left[\begin{array}{c}
e(t)-e\left(t_{k}\right) \\
\left(t-t_{k}\right) e\left(t_{k+1}\right) \\
\left(t-t_{k}\right) e\left(t_{k}\right) \\
\int_{t_{k}}^{t} e(\alpha) d \alpha
\end{array}\right]^{T} S_{1}(t)\left[\begin{array}{c}
-\dot{e}(t) \\
-e\left(t_{k}\right) \\
-e\left(t_{k+1}\right) \\
-e(t)
\end{array}\right]
\end{aligned}
$$$$
+2\left[\begin{array}{c}
\dot{e}(t) \\
e\left(t_{k+1}\right) \\
e\left(t_{k}\right) \\
e(t)
\end{array}\right]^{T} S_{1}(t)\left[\begin{array}{c}
e\left(t_{k+1}\right)-e(t) \\
\left(t_{k+1}-t\right) e\left(t_{k}\right) \\
\left(t_{k+1}-t\right) e\left(t_{k+1}\right) \\
\int_{t}^{t_{k+1}} e(\alpha) d \alpha
\end{array}\right]
$$$$
+2\left[\begin{array}{c}
e(t)-e\left(t_{k}\right) \\
\left(t-t_{k}\right) e\left(t_{k+1}\right) \\
\left(t-t_{k}\right) e\left(t_{k}\right) \\
\int_{t_{k}}^{t} e(\alpha) d \alpha
\end{array}\right]^{T}
$$$$
\cdot S_{1 d}\left[\begin{array}{c}
e\left(t_{k+1}\right)-e(t) \\
\left(t_{k+1}-t\right) e\left(t_{k}\right) \\
\left(t_{k+1}-t\right) e\left(t_{k+1}\right) \\
\int_{t}^{t_{k+1}} e(\alpha) d \alpha
\end{array}\right]=\operatorname{sym}\left\{\eta_{1} S_{1}(t) \eta_{2 d}^{T}\right.
$$$$
\left.+\eta_{1 d} S_{1}(t) \eta_{2}^{T}+\eta_{1} S_{1 d} \eta_{2}^{T}\right\}
$$$$
\dot{V}_{l 2}(t)=2\left[\begin{array}{c}
e(t)-e\left(t_{k}\right) \\
\int_{t_{k}}^{t} e(\alpha) d \alpha
\end{array}\right]^{T} S_{2}\left[\begin{array}{c}
-\dot{e}(t) \\
-e(t)
\end{array}\right]+2\left[\begin{array}{c}
\dot{e}(t) \\
e(t)
\end{array}\right]^{T}
$$$$
\cdot S_{2}\left[\begin{array}{c}
e\left(t_{k+1}\right)-e(t) \\
\int_{t}^{t_{k+1}} e(\alpha) d \alpha
\end{array}\right]
$$

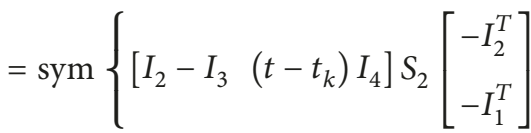$$
\left.+\left[\begin{array}{ll}
I_{2} & I_{1}
\end{array}\right] S_{2}\left[\begin{array}{c}
I_{5}^{T}-I_{1}^{T} \\
\left(t_{k+1}-t\right) I_{6}^{T}
\end{array}\right]\right\} \text {, }
$$$$
\dot{V}_{l 3}(t)=\left(t_{k+1}-t\right) \dot{e}^{T}(t) T_{1} e \dot{e}(t)-\int_{t_{k}}^{t} \dot{e}^{T}(\alpha)
$$$$
\cdot T_{1} \dot{e}(\alpha) d \alpha=\left(t_{k+1}-t\right) I_{2} T_{1} I_{2}^{T}-\int_{t_{k}}^{t} \dot{e}^{T}(\alpha)
$$

$\cdot T_{1} \dot{e}(\alpha) d \alpha$,

$$
\begin{gathered}
\dot{V}_{l 4}(t)=\left(t-t_{k}\right) \dot{e}^{T}(t) T_{2} \dot{e}(t)-\int_{t}^{t_{k+1}} \dot{e}^{T}(\alpha) \\
\cdot T_{2} \dot{e}(\alpha) d \alpha=\left(t-t_{k}\right) I_{2} T_{2} I_{2}^{T}-\int_{t}^{t_{k+1}} \dot{e}^{T}(\alpha)
\end{gathered}
$$

$\cdot T_{2} \dot{e}(\alpha) d \alpha$ 
Separating the integral in $\dot{V}_{2}$ for two sides and applying Lemmas 1 and 2, we have

$$
\begin{aligned}
& -\int_{t-\tau_{M}}^{t} \dot{e}^{T}(\alpha) R \dot{e}(\alpha) d \alpha \\
& =-\int_{t-\tau(t)}^{t} \dot{e}^{T}(\alpha) R \dot{e}(\alpha) d \alpha \\
& \quad-\int_{t-\tau_{M}}^{t-\tau(t)} \dot{e}^{T}(\alpha) R \dot{e}(\alpha) d \alpha
\end{aligned}
$$

$$
\begin{aligned}
& \leq-\zeta^{T}(t) \Sigma\left[\begin{array}{cc}
\widehat{R} & \widehat{M} \\
\widehat{M} & \widehat{R}
\end{array}\right] \Sigma^{T} \zeta(t) \\
& =-\zeta^{T}(t) \kappa_{4}\left[\begin{array}{cc}
\widehat{R} & \widehat{M} \\
\widehat{M} & \widehat{R}
\end{array}\right] \kappa_{4}^{T} \zeta(t)
\end{aligned}
$$

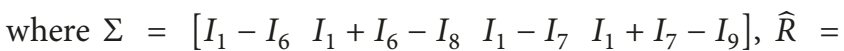
$\left[\begin{array}{ll}R & 0 \\ 0 & R\end{array}\right]$.

Using Lemma 3, the upper bound of each integrals in $\dot{V}_{l 3}(t), \dot{V}_{l 4}(t)$ is estimated as follows:

$$
\begin{aligned}
& -\int_{t_{k}}^{t} \dot{e}^{T}(\alpha) T_{1} \dot{e}(\alpha) d \alpha \leq \zeta^{T}(t)\left[\begin{array}{c}
\kappa_{1}^{T} \\
\kappa_{2}^{T}
\end{array}\right]^{T}\left[\begin{array}{cc}
\left(t-t_{k}\right) X_{1} T_{1}^{-1} X_{1}^{T} & X_{1}\left[\begin{array}{ll}
1 & 0
\end{array}\right] \\
* & \frac{\left(t-t_{k}\right)}{3} Y_{1} T_{1}^{-1} Y_{1}^{T}+\operatorname{sym}\left(Y_{1}\left[\begin{array}{ll}
-I & 2 I
\end{array}\right]\right)
\end{array}\right]\left[\begin{array}{c}
\kappa_{1}^{T} \\
\kappa_{2}^{T}
\end{array}\right] \zeta(t),
\end{aligned}
$$

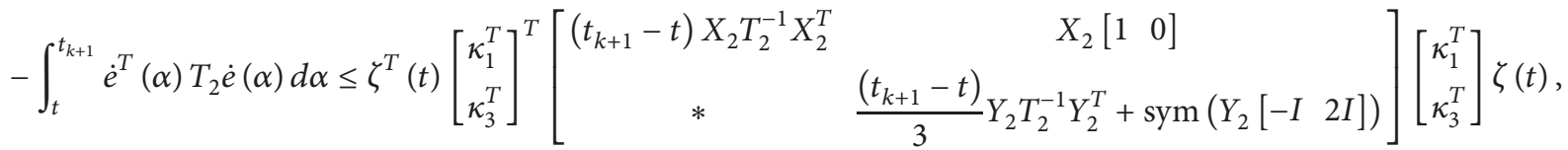

Then, the following is satisfied with the given nonlinear function as

$$
\begin{aligned}
& {\left[g\left(e_{i}(t), s_{i}(t)\right)-W_{1} e_{i}(t)\right]^{T}} \\
& \quad \cdot\left[g\left(e_{i}(t), s_{i}(t)\right)-W_{2} e_{i}(t)\right] \leq 0 .
\end{aligned}
$$

Equation (13) is equivalent to

$$
-\delta\left[\begin{array}{c}
e(t) \\
\widehat{g}(e(t), r(t))
\end{array}\right]^{T}\left[\begin{array}{cc}
\widetilde{W_{1}} & \widetilde{W_{2}} \\
* & 2 I
\end{array}\right]\left[\begin{array}{c}
e(t) \\
\widehat{g}(e(t), r(t))
\end{array}\right] \geq 0
$$

where $\widetilde{W_{1}}=\left(\mathrm{I} \otimes W_{1} W_{2}\right)+\left(W_{1} W_{2} \otimes \mathrm{I}\right), \widetilde{W_{2}}=-I \otimes\left(W_{1}^{T}+W_{2}^{T}\right)$, and $\delta$ is a constant scalar. Considering the dynamic equations (6) with auxiliary matrices $G_{1}, G_{2}, G_{3}, G_{4}$, the following holds:

$$
\begin{aligned}
& 2\left(\left(\left(\mathrm{t}-t_{k}\right) G_{1}+\left(t_{k+1}-t\right) G_{2}\right) I_{1}\right. \\
& \left.\quad+\left(\left(\mathrm{t}-t_{k}\right) G_{3}+\left(t_{k+1}-t\right) G_{4}\right) I_{2}\right) \cdot\left(c(C \otimes \mathrm{D}) I_{1}\right. \\
& \left.\quad+c(C \otimes \mathrm{A}) I_{7}+\mathrm{KI}_{3}-I_{2}+I_{11}\right)=0,
\end{aligned}
$$

Summing up (21), (24), (25), (27), and (28), the derivative of LKF is expressed as

$$
\dot{V}\left(x_{t}\right)=\zeta^{T}(t) \Lambda_{h(t)} \zeta(t)
$$

where

$$
\begin{aligned}
& \Lambda_{h(t)}=\Pi_{1, h(t)}+\left(\mathrm{t}-t_{k}\right) \Pi_{2}+\left(t_{k+1}-t\right) \Pi_{3} \\
& \Pi_{1, h(t)}=\operatorname{sym}\left\{I_{1} P I_{2}^{T}\right\}+I_{1} Q_{1} I_{1}^{T}-(1-\mu) I_{7} Q_{1} I_{7}^{T} \\
& +I_{1} Q_{2} I_{1}^{T}-I_{8} Q_{2} I_{8}^{T}+\tau_{M} I_{2} R I_{2}^{T}-\kappa_{4}\left[\begin{array}{cc}
\widehat{R} & \widehat{M} \\
\widehat{M} & \widehat{R}
\end{array}\right] \kappa_{4}^{T} \\
& +\operatorname{sym}\left\{\eta_{1} S_{1}(t) \eta_{2 d}^{T}+\eta_{1 d} S_{1}(t) \eta_{2}^{T}+\eta_{1} S_{1 d}(t) \eta_{2}^{T}\right\} \\
& +\operatorname{sym}\left\{\left[\begin{array}{ll}
I_{1}-I_{3} & \left(t-t_{k}\right) I_{4}
\end{array}\right] S_{2}\left[\begin{array}{c}
-I_{2}^{T} \\
-I_{1}^{T}
\end{array}\right]\right. \\
& \left.+\left[\begin{array}{ll}
I_{2} & I_{1}
\end{array}\right] S_{2}\left[\begin{array}{c}
I_{5}^{T}-I_{1}^{T} \\
\left(t_{k+1}-t\right) I_{6}^{T}
\end{array}\right]\right\}+\left(t_{k+1}-t\right) I_{2} T_{1} I_{2}^{T} \\
& +\left(t-t_{k}\right) I_{2} T_{2} I_{2}^{T}+\operatorname{sym}\left\{\kappa_{1} X_{1}\left[\begin{array}{ll}
I & 0
\end{array}\right] \kappa_{2}^{T}\right. \\
& \left.+\kappa_{2} Y_{1}\left[\begin{array}{ll}
-I & 2 I
\end{array}\right] \kappa_{2}^{T}\right\}+\operatorname{sym}\left\{\kappa_{1} X_{2}\left[\begin{array}{ll}
I & 0
\end{array}\right] \kappa_{3}^{T}\right. \\
& \left.+\kappa_{1} Y_{2}\left[\begin{array}{ll}
-I & 2 I
\end{array}\right] \kappa_{3}^{T}\right\}-\delta\left[\begin{array}{ll}
I_{1} & I_{11}
\end{array}\right]\left[\begin{array}{cc}
\widetilde{W}_{1} & \widetilde{W}_{2} \\
* & 2 I
\end{array}\right]\left[\begin{array}{c}
I_{1}^{T} \\
I_{11}^{T}
\end{array}\right] \\
& +\operatorname{sym}\left\{\left(I_{1} G_{1}+I_{2} G_{2}\right)\right. \\
& \left.\cdot\left(c(C \otimes D) I_{1}^{T}+c(C \otimes A) I_{7}^{T}+K I_{3}^{T}-I_{2}^{T}+I_{11}^{T}\right)\right\} \\
& \Pi_{2}=\kappa_{1} X_{1} T_{1}^{-1} X_{1}^{T} \kappa_{1}^{T}+\frac{1}{3} \kappa_{2} Y_{1} T_{1}^{-1} Y_{1}^{T} \kappa_{2}^{T} \\
& \Pi_{3}=\kappa_{1} X_{2} T_{2}^{-1} X_{2}^{T} \kappa_{1}^{T}+\frac{1}{3} \kappa_{3} Y_{2} T_{2}^{-1} Y_{2}^{T} \kappa_{3}^{T}
\end{aligned}
$$


Therefore, the conditions $\zeta^{T}(t) \Lambda_{h(t)} \zeta(t)<0$ implies that $\dot{V}<$ 0 for $t \in\left[t_{k}, t_{k+1}\right)$. From the convex combination method, $\Lambda_{h(t)}<0$ for $h(t) \in\left[0, h_{M}\right)$ is equivalent to the conditions in Theorem 6 using Schur complements, and thus the synchronization error system is asymptotically stable for given sampling time $h_{M}$. This is the end of the proof.

Remark 7. The constructed Lyapunov functionals include novel looped functionals, which are $\mathrm{V}_{l 1}, \mathrm{~V}_{l 2}, \mathrm{~V}_{l 3}$, and $\mathrm{V}_{l 4}$. Those functionals satisfy $\mathrm{V}_{l 1}=\mathrm{V}_{l 2}=\mathrm{V}_{l 3}=0$ at every sampling instances $t_{k}, t_{k+1}$ so it is looped functional as defined in [23].

Remark 8. The additional consideration of $t_{k+1}$ in LyapunovKrasovskii functional (LKF) results in deriving less conservative results, which reflect more information for sampling time. The idea is motivated by the two-sided loopedfunctional [24], which is applied to CDNs with timevarying coupling delay to achieve more stable synchronization between two or more nodes. In the stability analysis of the sampled-data system using looped-functional, it is important to take into account the sampled-time-dependent vectors which become zero at each end point of each sampling time. However, in [24], the product term between the vectors $\mathrm{x}(\mathrm{t})-\mathrm{x}\left(t_{k}\right)$ and $\mathrm{x}\left(t_{k+1}\right)-\mathrm{x}(\mathrm{t})$ and the product term between $x\left(t_{k}\right)$ and $x\left(t_{k+1}\right)$ are separately considered. In this paper, more generalized results are derived by defining the vector as $\eta$ which provides each cross terms at the same time.

Remark 9. The novelty of the proposed looped functional is in the formation of $V_{l 1}$. In $V_{l 1}$, the variables are chosen as $\eta_{1}, \eta_{2}$ and $S(t)$ is composed of sampled-time-dependent matrix. The dimension of the LMI variable $S_{1}(t)$ is extended to $R^{4 \times 4}$. Therefore, augmented vectors provide an increased degrees of freedom (DoF) and improved results compared with the existing results. Herein, the DoF is the number of independent variables or equations that must be specified to solve the problem uniquely. It extends the range of the solution and provides less conservative conditions.

Remark 10. In Theorem 6, a sufficient condition for the synchronization is derived in terms of LMIs which is obtained by constructing new looped functional. The results are sufficient conditions, which imply that there is still room for further improvement. Some approaches to reducing the conservatism are available. The conservativeness will be reduced by augmented vector or segmenting formulas. Also, new Lyapunov functions such as Lyapunov-Krasovskii or discontinuous Lyapunov [30] may play an essential role in the further reduction of the conservativeness.

Based on Theorem 6, the following corollary is constructed for the stabilization problem.

Corollary 11. For given scalars $\alpha, 0 \leq \beta \leq 1$, and $h_{M}$, if there exist a positive scalar $\delta$, symmetric matrices $P>0, Q_{1}>0$, $Q_{2}>0, R>0, T_{1}>0$, and $T_{2}>0$, and any matrices $S_{1}(t)$, $S_{2}, X_{1}, X_{2}, Y_{1}, Y_{2}, \widehat{M}, H_{a}, H_{b}, G_{a}=\operatorname{diag}\left\{G_{a 1}, G_{a 2}, \ldots, G_{a n}\right\}$, $G_{b}=\operatorname{diag}\left\{G_{b 1}, G_{b 2}, \ldots, G_{b n}\right\}$,

$$
\begin{aligned}
& {\left[\begin{array}{ccc}
\widehat{\boldsymbol{\Pi}}_{1, \boldsymbol{h}(\boldsymbol{t})=\mathbf{0}} & \kappa_{1} X_{2} & \kappa_{3} Y_{2} \\
* & -h_{M} T_{2} & 0 \\
* & 0 & -3 h_{M} T_{2}
\end{array}\right] \leq 0,} \\
& {\left[\begin{array}{ccc}
\widehat{\boldsymbol{\Pi}}_{1, \boldsymbol{h}(\boldsymbol{t})=\mathbf{h}} & \kappa_{1} X_{1} & \kappa_{2} Y_{1} \\
* & -h_{M} T_{1} & 0 \\
* & 0 & -3 h_{M} T_{1}
\end{array}\right] \leq 0,} \\
& \\
& {\left[\begin{array}{cc}
\widehat{R} & \widehat{M} \\
\widehat{M} & \widehat{R}
\end{array}\right] \geq 0,}
\end{aligned}
$$

where

$$
\begin{aligned}
& \widehat{\boldsymbol{\Pi}}_{\mathbf{1}, \boldsymbol{h}(\boldsymbol{t})}=\operatorname{sym}\left\{I_{1} P_{1} I_{2}^{T}\right\}+I_{1} Q_{1} I_{1}^{T}-(1-\mu) I_{7} Q_{1} I_{7}^{T} \\
& +I_{1} Q_{2} I_{1}^{T}-I_{8} Q_{2} I_{8}^{T}+\tau_{M} I_{2} R I_{2}^{T}-\kappa_{4}\left[\begin{array}{cc}
\widehat{R} & \widehat{M} \\
\widehat{M} & \widehat{R}
\end{array}\right] \kappa_{4}^{T} \\
& +\operatorname{sym}\left\{\eta_{1} S_{1}(t) \eta_{2 d}^{T}+\eta_{1 d} S_{1}(t) \eta_{2}^{T}+\eta_{1} S_{1 d}(t) \eta_{2}^{T}\right\} \\
& +\operatorname{sym}\left\{\left[\begin{array}{ll}
I_{1}-I_{3} & \left(t-t_{k}\right) I_{4}
\end{array}\right] S_{2}\left[\begin{array}{c}
-I_{2}^{T} \\
-I_{1}^{T}
\end{array}\right]\right. \\
& \left.+\left[\begin{array}{ll}
I_{2} & I_{1}
\end{array}\right] S_{2}\left[\begin{array}{c}
I_{5}^{T}-I_{1}^{T} \\
\left(t_{k+1}-t\right) I_{6}^{T}
\end{array}\right]\right\}+\left(t_{k+1}-t\right) I_{2} T_{1} I_{2}^{T} \\
& +\left(t-t_{k}\right) I_{2} T_{2} I_{2}^{T}+\operatorname{sym}\left\{\kappa_{1} X_{1}\left[\begin{array}{ll}
I & 0
\end{array}\right] \kappa_{2}^{T}\right. \\
& \left.+\kappa_{2} Y_{1}\left[\begin{array}{ll}
-I & 2 I
\end{array}\right] \kappa_{2}^{T}\right\}+\operatorname{sym}\left\{\kappa_{1} X_{2}\left[\begin{array}{ll}
I & 0
\end{array}\right] \kappa_{3}^{T}\right. \\
& \left.+\kappa_{1} Y_{2}\left[\begin{array}{ll}
-I & 2 I
\end{array}\right] \kappa_{3}^{T}\right\}-\delta\left[\begin{array}{ll}
I_{1} & I_{11}
\end{array}\right]\left[\begin{array}{cc}
\widetilde{W}_{1} & \widetilde{W}_{2} \\
* & 2 I
\end{array}\right]\left[\begin{array}{c}
I_{1}^{T} \\
I_{11}^{T}
\end{array}\right] \\
& +\left(t-t_{k}\right) \operatorname{sym}\left\{( \beta _ { 1 } I _ { 1 } + \alpha I _ { 2 } ) \cdot \left(G_{a} I_{11}^{T}\right.\right. \\
& +c G_{a}(C \otimes D) I_{1}^{T}+c G_{a}(C \otimes A) I_{7}^{T}+H_{a} I_{3}^{T} \\
& \left.\left.-G I_{2}^{T}\right)\right\}+\left(t_{k+1}-t\right) \operatorname{sym}\left\{( \beta _ { 2 } I _ { 1 } + \alpha I _ { 2 } ) \cdot \left(G_{a} I_{11}^{T}\right.\right. \\
& +c G_{b}(C \otimes D) I_{1}^{T}+c G_{b}(C \otimes A) I_{7}^{T}+H_{b} I_{3}^{T} \\
& \left.\left.-G_{b} I_{2}^{T}\right)\right\}
\end{aligned}
$$

then the control gain is given by $\mathrm{h}=G_{a}^{-1} H_{a}$ which stabilizes the error dynamics.

Proof. Substituting the variables $G_{1}, G_{2}, G_{3}$, and $G_{4}$ in the zero equation (28) into $\beta_{1} G_{a}, \beta_{2} G_{b}, \alpha G_{a}$, and $\alpha G_{b}$, respectively, the following are obtained:

$$
\begin{aligned}
& 2\left(t-t_{k}\right)\left[( \beta _ { 1 } I _ { 1 } + \alpha I _ { 2 } ) \cdot \left(c G_{a}(C \otimes D) I_{1}^{T}\right.\right. \\
& \left.\left.\quad+c G_{a}(C \otimes A) I_{7}^{T}+H_{a} I_{3}^{T}-G_{a} I_{2}^{T}+G_{a} I_{11}^{T}\right)\right]=0,
\end{aligned}
$$




$$
\begin{aligned}
& 2\left(t_{k+1}-t\right)\left[( \beta _ { 2 } I _ { 1 } + \alpha I _ { 2 } ) \cdot \left(c G_{b}(C \otimes D) I_{1}^{T}\right.\right. \\
& \left.\left.\quad+c G_{b}(C \otimes A) I_{7}^{T}+H_{b} I_{3}^{T}-G_{b} I_{2}^{T}+G_{b} I_{11}^{T}\right)\right]=0 .
\end{aligned}
$$

where $H_{a}=G_{a} K$ and $H_{b}=G_{b} K$. The other part of the proof is omitted for brevity as it is similar to that of Theorem 6 .

\section{Numerical Examples}

In this section, two examples are revisited from literature [5].

Example 12. Let us consider the CDNs composed of 3 nodes with the following matrices and parameters:

$$
\begin{aligned}
A & =\left[\begin{array}{ll}
1 & 0 \\
0 & 1
\end{array}\right], \\
D & =\left[\begin{array}{ll}
0 & 0 \\
0 & 0
\end{array}\right], \\
G & =\left[\begin{array}{lll}
-1 & 0 & 1 \\
0 & -1 & 1 \\
1 & 1 & 2
\end{array}\right], \\
c & =0.5, \\
\tau_{M} & =0.5, \\
\mu & =0.25 .
\end{aligned}
$$

The nonlinear function is defined as

$$
f\left(x_{i}(t)\right)=\left[\begin{array}{c}
-0.5 x_{i 1}+\tanh \left(0.2 x_{i 1}\right)+0.2 x_{i 1} \\
0.95 x_{i 2}-\tanh \left(0.75 x_{i 2}\right)
\end{array}\right],
$$

satisfying the condition in (3) by the following matrices:

$$
\begin{aligned}
& W_{1}=\left[\begin{array}{cc}
-0.5 & 0.2 \\
0 & 0.95
\end{array}\right], \\
& W_{2}=\left[\begin{array}{cc}
-0.3 & 0.2 \\
0 & 0.2
\end{array}\right] .
\end{aligned}
$$

For $\alpha=1.2, h_{M}=2.01$, the corresponding gain matrices are given by

$$
\begin{aligned}
& K_{1}=\left[\begin{array}{cc}
0.1117 & -0.1514 \\
-0.0761 & -0.8361
\end{array}\right], \\
& K_{2}=\left[\begin{array}{cc}
0.1117 & -0.1514 \\
-0.0761 & -0.8361
\end{array}\right], \\
& K_{3}=\left[\begin{array}{cc}
0.2682 & -0.1716 \\
-0.0949 & -0.8335
\end{array}\right] .
\end{aligned}
$$

$K$ are designed using the toolbox YALMIP 3.0 and SeDuMi 1.3 of MATLAB. The time-varying delay is chosen as $\tau(t)=$ $0.25+0.1 \sin ^{2}(5 t)$. The initial state is $x(0)=[4,-3,-2,1,2,-5]^{T}$.

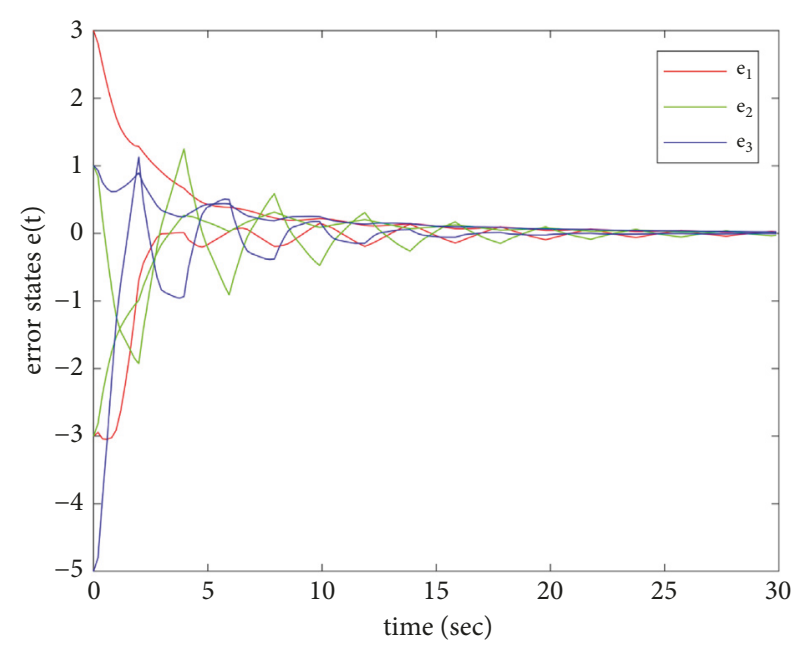

FIgURE 1: The state trajectories of the system (38) with $c=0.5, \tau_{M}=$ 0.5 , and $\mu=0.25$.

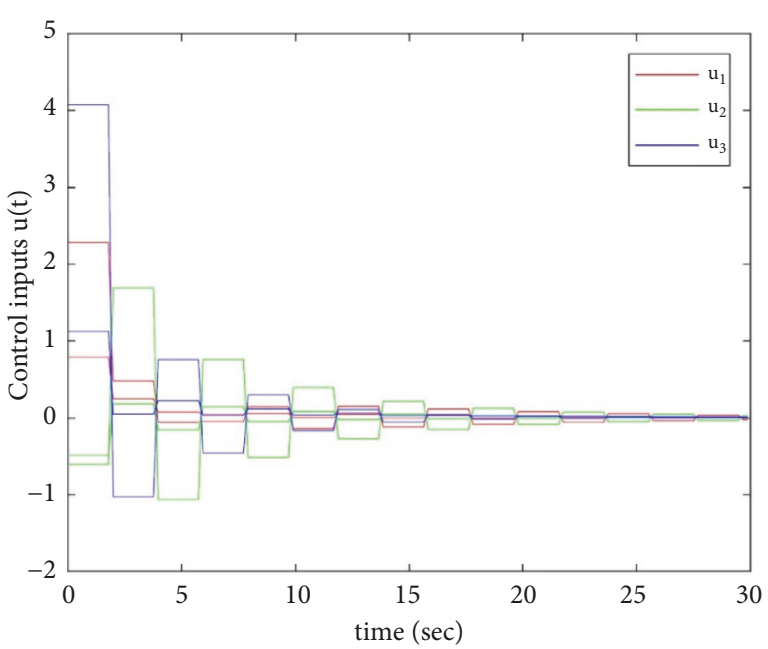

FIgURE 2: The input signals of the system (38) with $c=0.5, \tau_{M}=0.5$, and $\mu=0.25$.

The state trajectories of error system and the sampleddata input signals are, respectively, presented in Figures 1 and 2, which show stable convergence. Figure 1 shows the trajectories of error dynamics which is controlled by using the gain matrices at the simulation time from 0 to 30 seconds. The input signals $\left(\mathrm{u}_{1}, \mathrm{u}_{2}\right.$, and $\left.\mathrm{u}_{3}\right)$ converge, respectively, to 0 in Figure 2.

Example 13. Consider Chua's circuit composed of 4 nodes. The dynamics of Chua's circuit is represented as

$$
\begin{aligned}
& \dot{x}_{1}(t)=\epsilon_{1}\left(-x_{1}(t)+x_{2}(t)-h\left(x_{1}(t)\right)\right) \\
& \dot{x}_{2}(t)=-x_{1}(t)-x_{2}(t)+x_{3}(t) \\
& \dot{x}_{3}(t)=-\epsilon_{2} x_{2}(t)
\end{aligned}
$$

where $\mathrm{h}\left(x_{1}(t)\right)=m_{2} x_{1}(t)+0.5\left(m_{1}+m_{2}\right)\left(\left\|x_{1}(t)+1\right\|-\| x_{1}(t)-\right.$ $1 \|)$ with the parameters $\epsilon_{1}=10, \epsilon_{2}=14.87, m_{1}=-1.27$, 
and $m_{2}=-0.68$. Then, the nonlinear function is denoted as

$$
\begin{aligned}
& f\left(x_{i}(t)\right) \\
& =\left[\begin{array}{ccc}
-\epsilon_{1}\left(1+m_{2}\right) & \epsilon_{1} & 0 \\
1 & -1 & 1 \\
0 & -\epsilon_{2} & 0
\end{array}\right] x_{i}(t) \\
& +\left[\begin{array}{cc}
-\epsilon_{1}\left(m_{1}+m_{2}\right)\left(\left\|x_{i 1}(t)+1\right\|-\left\|x_{i 1}(t)-1\right\|\right) \\
0 \\
0
\end{array}\right]
\end{aligned}
$$

From (4), the matrices are given as

$$
\begin{aligned}
& W_{1}=\left[\begin{array}{ccc}
2.7 & 10 & 0 \\
1 & -1 & 0 \\
0 & -14.87 & 0
\end{array}\right], \\
& W_{2}=\left[\begin{array}{ccc}
-3.2 & 10 & 0 \\
1 & -1 & 1 \\
0 & -14.87 & 0
\end{array}\right] .
\end{aligned}
$$

For given systems, the inner coupling matrix $A$, the outer coupling $D$, and parameters are chosen as

$$
\begin{aligned}
A & =I_{N}, \\
D & =0_{n} \\
G & =\left[\begin{array}{cccc}
-3 & 1 & 1 & 1 \\
1 & -2 & 1 & 0 \\
1 & 1 & -2 & 0 \\
1 & 0 & 0 & -1
\end{array}\right], \\
c & =0.5, \\
\tau_{M} & =0.5 \\
\mu & =0.1 .
\end{aligned}
$$

The controller gain of Example 13 was calculated using toolbox YALMIP 3.0 and SeDuMi 1.3 of MATLAB in the same manner of Example 12. The corresponding gain with $\alpha=0.7$, $h_{M}=0.2138$ is given as

$$
\begin{aligned}
& K_{1}=\left[\begin{array}{ccc}
-6.7314 & -4.1356 & 1.0795 \\
-0.0834 & -1.1048 & -0.7895 \\
6.6711 & 7.6457 & -2.1652
\end{array}\right], \\
& K_{2}=\left[\begin{array}{ccc}
-7.7827 & -3.5618 & 1.0721 \\
-0.1367 & -2.0333 & -0.5521 \\
6.6458 & 7.3950 & -3.0248
\end{array}\right],
\end{aligned}
$$

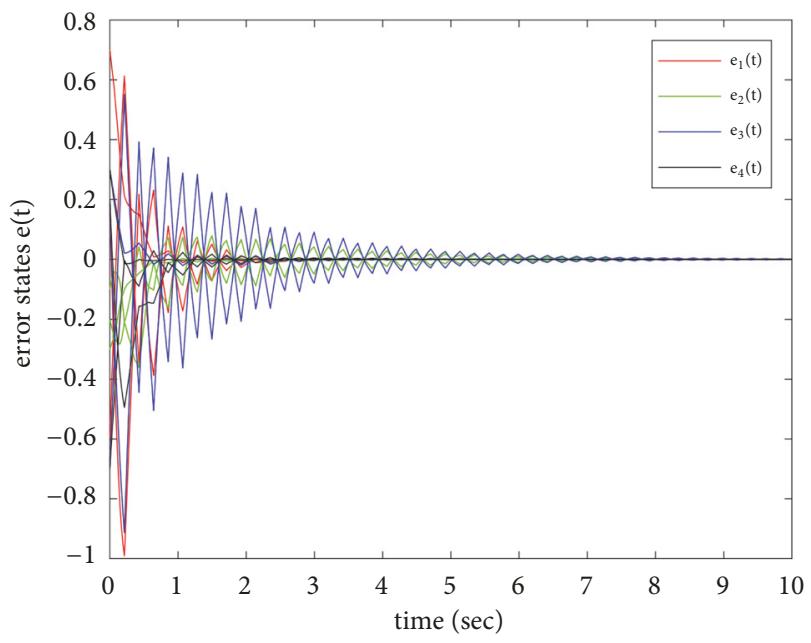

FIGURE 3: The error trajectories of system (41) with $c=0.5, \tau_{M}=0.5$, and $\mu=0.1$.

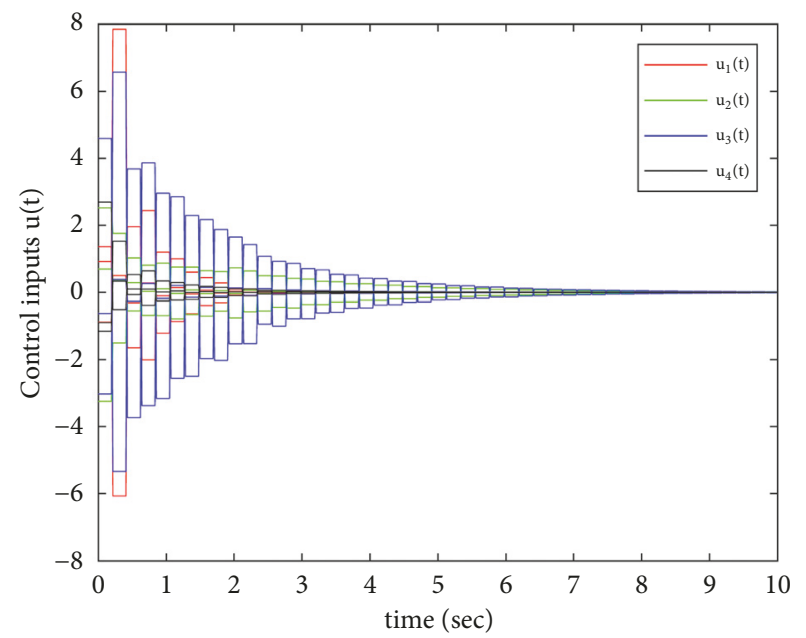

FIgURE 4: The sampled-data input signals of system (41) with $c=0.5$, $\tau_{M}=0.5$, and $\mu=0.1$.

$$
\begin{aligned}
& K_{3}=\left[\begin{array}{ccc}
-7.7821 & -3.5679 & 1.0678 \\
-0.1372 & -2.0340 & -0.5508 \\
6.6346 & 7.4183 & -3.0214
\end{array}\right], \\
& K_{4}=\left[\begin{array}{ccc}
-7.8447 & -3.4030 & 1.1874 \\
-0.0468 & -2.3492 & -0.5990 \\
6.4107 & 7.2538 & -3.3484
\end{array}\right] .
\end{aligned}
$$

The trajectories of error dynamics and the sampled-data input signals are represented in Figures 3 and 4, respectively.

The maximum allowable sampling time is computed; the result using Corollary 11 is compared with existing results in Table 1. The results show that Corollary 11 provides more considerable maximum sampling period. It means that the synchronized error system guarantees stability in the broader 
TABLE 1: The allowable maximum sampling time for Examples 12 and 13.

\begin{tabular}{lccccc}
\hline & ref. [5] & ref. [31] & ref. [16] & ref. [19] & Corollary 11 \\
\hline$h_{M}$ & 0.5409 & 0.5573 & 0.9016 & 1.3756 & 1.9789 \\
\hline$h_{M}$ & 0.0790 & 0.0793 & 0.1607 & 0.1833 & 0.2138 \\
\hline Nov & $3 \iota^{2}+(4+n) \iota+1$ & $3.5 \iota^{2}+3.5 \iota+1$ & $11.5 \iota^{2}+3.5 \iota+1$ & $7 \iota^{2}+(4+n) \iota+1$ & $34 \iota^{2}+5 \iota+1$ \\
\hline
\end{tabular}

sampling region. The last line of Table 1 represents a number of decision variables (NoV). It can be expressed as the product of the number of node and dimension of the node, which is defined as $\iota=\mathrm{N} \times \mathrm{n}$. Our method has higher complexity due to a larger NoV than ones of other methods. However, the proposed method is efficient on offline work with a more considerable allowable maximum sampling time.

\section{Conclusions}

This paper provides the new stabilization criteria to increase the maximum sampling interval for the synchronization of CDN with time-varying coupling delay. Novel two-sided looped functional and QGFWI are presented to obtain the enhanced results. The two-sided stabilization method is formulated by additional free matrices for present and next sampling time. The proposed looped functional, which vanishes at current sampling time and the next one, is constructed by using the augmented states to consider the information of sawtooth shape sampling pattern. Finally, simulation results show that the proposed synchronization approach provides a larger maximum sampling interval than one of the existing results. In other words, the proposed method contributes to extending the stable region and deriving a less conservative result. Also, the effectiveness of the proposed sampleddata control scheme has been demonstrated by numerical examples.

In future work, practical situations will be considered in sampled-data control for CDNs. For example, the proposed scheme could be applied to the synchronization of CDN systems with heterogeneous time-varying delay or with asynchronous and aperiodic sampling characteristics.

\section{Data Availability}

The simulation result data used to support the findings of this study are included within the article.

\section{Conflicts of Interest}

The authors declare that there are no conflicts of interest regarding the publication of this paper.

\section{Acknowledgments}

This work was supported by Electronics and Telecommunications Research Institute (ETRI) grant funded by the Korean Government (18ZD1120, Support Project for Advanced ICT Convergence Technology based on Regional Industry) and Basic Science Research Program through the National Research Foundation of Korea (NRF) funded by the Ministry of Education, Science and Technology (2016R1D1A1B03930623).

\section{References}

[1] A. Arenas, A. Díaz-Guilera, and C. J. Pérez-Vicente, "Synchronization processes in complex networks," Physica D: Nonlinear Phenomena, vol. 224, no. 1-2, pp. 27-34, 2006.

[2] M. Yuan, W. Wang, X. Luo, L. Liu, and W. Zhao, "Finite-time anti-synchronization of memristive stochastic BAM neural networks with probabilistic time-varying delays," Chaos, Solitons \& Fractals, vol. 113, pp. 244-260, 2018.

[3] M. Yuan, X. Luo, W. Wang, L. Li, and H. Peng, "Pinning synchronization of coupled memristive recurrent neural networks with mixed time-varying delays and perturbations," Neural Processing Letters, 2018.

[4] M. Yuan, W. Wang, X. Luo et al., "Synchronization of a class of memristive stochastic bidirectional associative memory neural networks with mixed time-varying delays via sampleddata control," Mathematical Problems in Engineering, vol. 2018, Article ID 9126183, 24 pages, 2018.

[5] N. Li, Y. Zhang, J. Hu, and Z. Nie, "Synchronization for general complex dynamical networks with sampled-data," Neurocomputing, vol. 74, no. 5, pp. 805-811, 2011.

[6] Z. Li and G. Chen, "Global synchronization and asymptotic stability of complex dynamical networks," IEEE Transactions on Circuits and Systems II: Express Briefs, vol. 53, no. 1, pp. 28-33, 2006.

[7] B. Wang and Z.-H. Guan, "Chaos synchronization in general complex dynamical networks with coupling delays," Nonlinear Analysis: Real World Applications, vol. 11, no. 3, pp. 1925-1932, 2010.

[8] C. P. Li, W. G. Sun, and J. Kurths, "Synchronization of complex dynamical networks with time delays," Physica A: Statistical Mechanics and its Applications, vol. 361, no. 1, pp. 24-34, 2006.

[9] J. Lu and G. Chen, "A time-varying complex dynamical network model and its controlled synchronization criteria," IEEE Transactions on Automatic Control, vol. 50, no. 6, pp. 841-846, 2005.

[10] W. Yu, G. Chen, and J. Lv, "On pinning synchronization of complex dynamical networks," Automatica, vol. 45, no. 2, pp. 429-435, 2009.

[11] Z.-H. Guan, Z.-W. Liu, G. Feng, and Y.-W. Wang, "Synchronization of complex dynamical networks with time-varying delays via impulsive distributed control," IEEE Transactions on Circuits and Systems I: Regular Papers, vol. 57, no. 8, pp. 2182-2195, 2010.

[12] X. Yang, J. Cao, and J. Lu, "Stochastic synchronization of complex networks with nonidentical nodes via hybrid adaptive and impulsive control," IEEE Transactions on Circuits and Systems I: Regular Papers, vol. 59, no. 2, pp. 371-384, 2012.

[13] S. Tong, S. Sui, and Y. Li, "Fuzzy adaptive output feedback control of MIMO nonlinear systems with partial tracking errors constrained," IEEE Transactions on Fuzzy Systems, vol. 23, no. 4, pp. 729-742, 2014. 
[14] L. Su and H. Shen, "Mixed Ho/passive synchronization for complex dynamical networks with sampled-data control," Applied Mathematics and Computation, vol. 259, pp. 931-942, 2015.

[15] L. Su, D. Ye, and X. Yang, "Dissipative-based sampled-data synchronization control for complex dynamical networks with time-varying delay," Journal of The Franklin Institute, vol. 354, no. 15, pp. 6855-6876, 2017.

[16] Z.-G. Wu, P. Shi, H. Su, and J. Chu, "Sampled-Data exponential synchronization of complex dynamical networks with timevarying coupling delay," IEEE Transactions on Neural Networks and Learning Systems, vol. 24, no. 8, pp. 1177-1187, 2013.

[17] W. Wang, M. Yu, X. Luo, L. Liu, M. Yuan, and W. Zhao, "Synchronization of memristive BAM neural networks with leakage delay and additive time-varying delay components via sampled-data control," Chaos, Solitons \& Fractals, vol. 104, pp. 84-97, 2017.

[18] W. Wang, X. Yu, X. Luo, and J. Kurths, "Synchronization control of memristive multidirectional associative memory neural networks and applications in network security communication," IEEE Access, vol. 6, pp. 36002-36018, 2018.

[19] Y. Liu and S. M. Lee, "Improved results on sampled-data synchronization of complex dynamical networks with timevarying coupling delay," Nonlinear Dynamics, vol. 81, no. 1-2, pp. 931-938, 2015.

[20] X. Wang, K. She, S. Zhong, and H. Yang, "New result on synchronization of complex dynamical networks with time-varying coupling delay and sampled-data control," Neurocomputing, vol. 214, pp. 508-515, 2016.

[21] Z. Chen, K. Shi, and S. Zhong, "New synchronization criteria for complex delayed dynamical networks with sampled-data feedback control," ISA Transactions ${ }^{\circledR}$, vol. 63, pp. 154-169, 2016.

[22] S. H. Lee, M. J. Park, O. M. Kwon, and R. Sakthivel, "Advanced sampled-data synchronization control for complex dynamical networks with coupling time-varying delays," Information Sciences, vol. 420, pp. 454-465, 2017.

[23] C. Briat and A. Seuret, "A looped-functional approach for robust stability analysis of linear impulsive systems," Systems \& Control Letters, vol. 61, no. 10, pp. 980-988, 2012.

[24] H.-B. Zeng, K. L. Teo, and Y. He, "A new looped-functional for stability analysis of sampled-data systems," Automatica, vol. 82, pp. 328-331, 2017.

[25] A. Seuret and F. Gouaisbaut, "Wirtinger-based integral inequality: application to time-delay systems," Automatica, vol. 49, no. 9, pp. 2860-2866, 2013.

[26] P. G. Park, J. W. Ko, and C. Jeong, "Reciprocally convex approach to stability of systems with time-varying delays," Automatica, vol. 47, no. 1, pp. 235-238, 2011.

[27] H.-B. Zeng, Y. He, M. Wu, and S.-P. Xiao, "Stability analysis of generalized neural networks with time-varying delays via a new integral inequality," Neurocomputing, vol. 161, pp. 148-154, 2015.

[28] D. Zhang, Q.-L. Han, and X. Jia, "Network-based output tracking control for T-S fuzzy systems using an event-triggered communication scheme," Fuzzy Sets and Systems, vol. 273, pp. 2648, 2015.

[29] C. Peng, Q.-L. Han, and D. Yue, "To transmit or not to transmit: a discrete event-triggered communication scheme for networked takagi-sugeno fuzzy systems," IEEE Transactions on Fuzzy Systems, vol. 21, no. 1, pp. 164-170, 2013.

[30] N. Eghbal, N. Pariz, and A. Karimpour, "Discontinuous piecewise quadratic Lyapunov functions for planar piecewise affine systems," Journal of Mathematical Analysis and Applications, vol. 399, no. 2, pp. 586-593, 2013.
[31] Z. Wu, J. H. Park, H. Su, B. Song, and J. Chu, "Exponential synchronization for complex dynamical networks with sampleddata," Journal of The Franklin Institute, vol. 349, no. 9, pp. 27352749, 2012. 


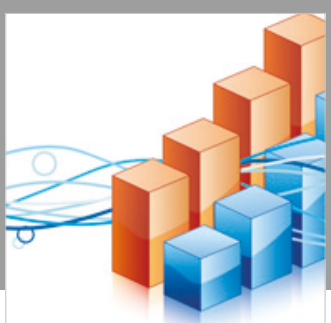

Advances in

Operations Research

\section{-n-m}
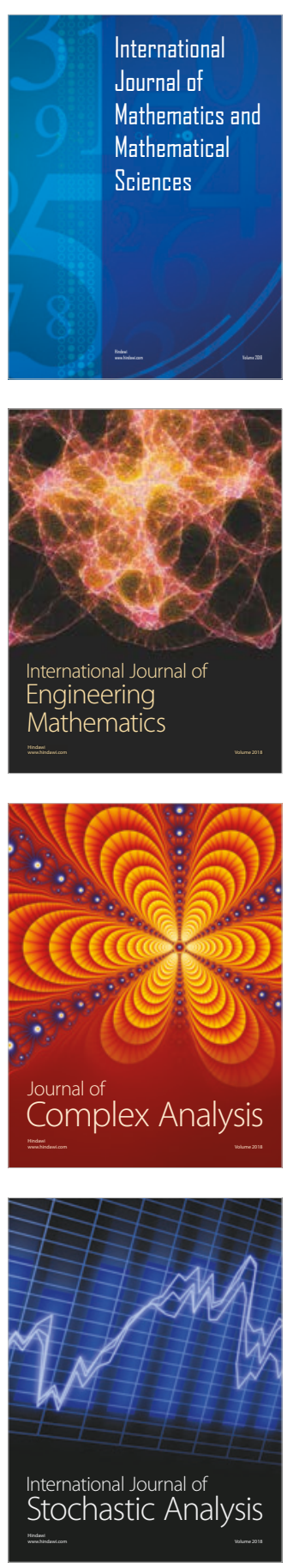
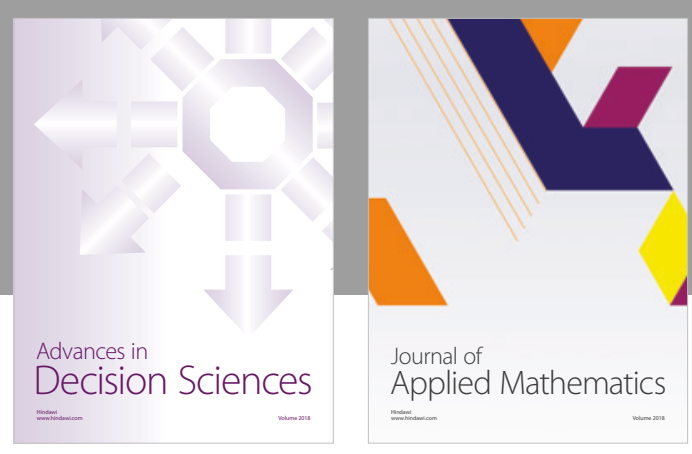

Journal of

Applied Mathematics
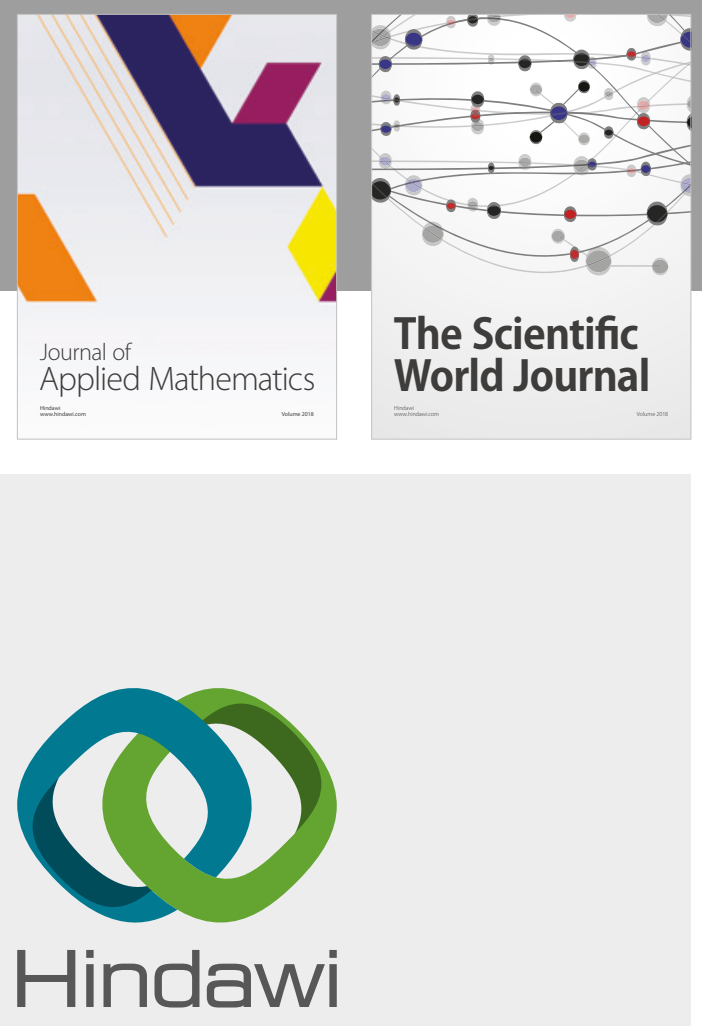

Submit your manuscripts at

www.hindawi.com

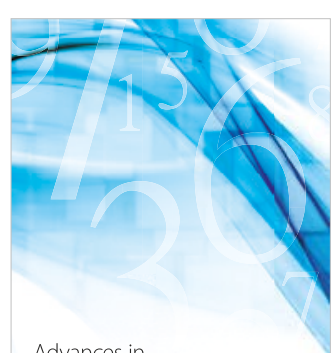

Advances in
Numerical Analysis
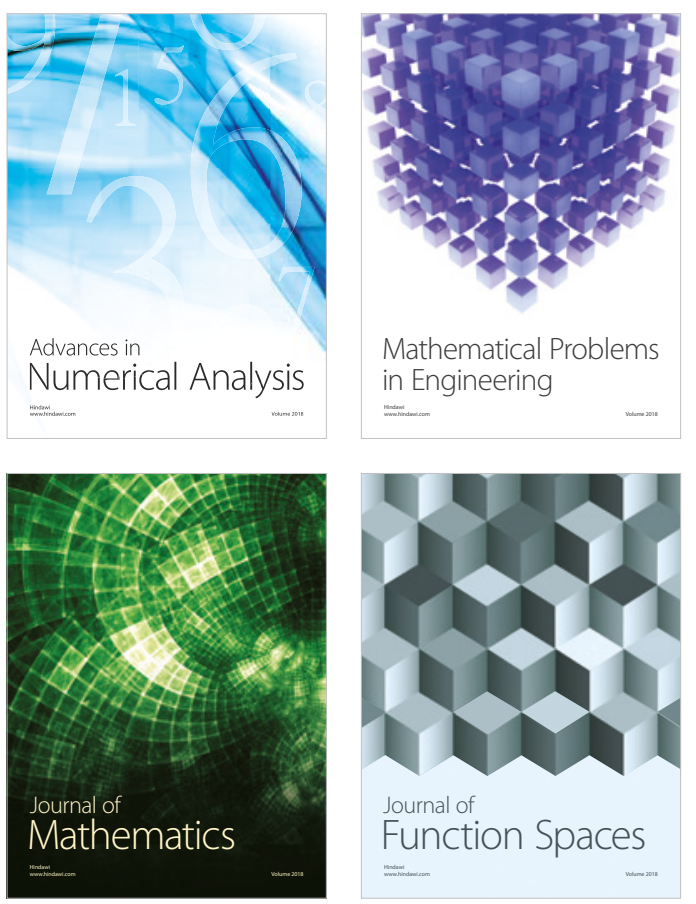

Mathematical Problems in Engineering

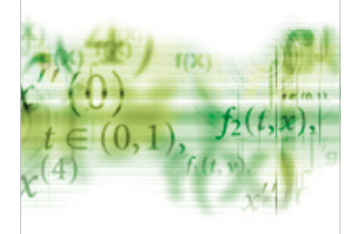

International Journal of

Differential Equations

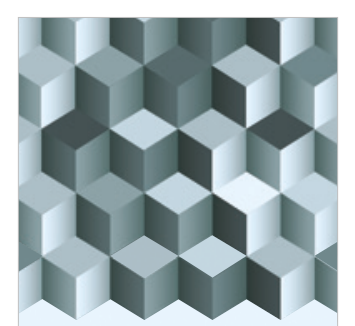

Journal of

Function Spaces

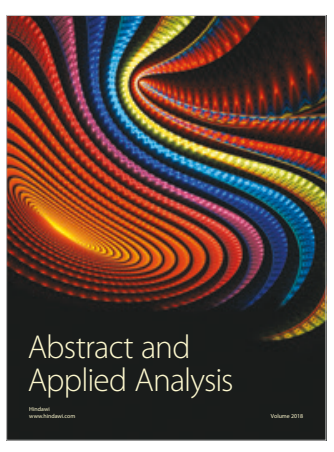

The Scientific

World Journal

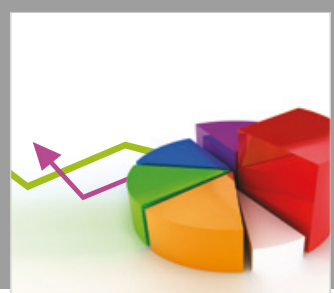

Journal of

Probability and Statistics
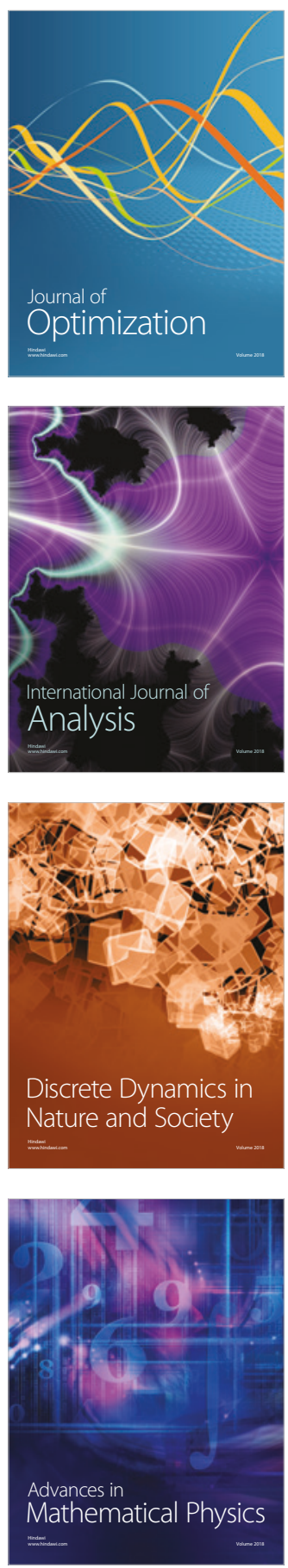